\title{
Estado de la industria de alta precisión en Costa Rica
}

Overview of the high precision industry in Costa Rica

Natalia Robles-Obando'

Fecha de recepción: 21 de enero del 2013 Fecha de aprobación: 26 de mayo del 2013

Robles, N. Estado de la industria de alta precisión en Costa Rica. Tecnología en Marcha. Vol. 26, Nº 4. Pág 85-91 


\section{Palabras clave}

Prácticas del maquinado; industria del maquinado.

\section{Resumen}

Esta investigación de las prácticas actuales en la manufactura de partes de ensambles o acoples en Costa Rica se fundamentó en el estudio de las prácticas de empresas representativas a nivel nacional. El objetivo fue conocer las prácticas de manufactura del sector y determinar sus necesidades de investigación y apoyo. Los resultados se cruzaron con las prácticas de una empresa internacional, así como otras fuentes de información del sector.

La mayoría de los casos estudiados coincide en que aún hay debilidades en los planos pues muchas veces carecen de la concepción del funcionamiento final de la parte o de los requerimientos para su acople. Coinciden también en la necesidad de tener más dominio en la asignación de tolerancias a las partes. En los casos nacionales se encontró que la labor del diseño recae en muy pocas personas.

En el estudio se evidenció claramente una contracción importante del sector durante la reciente crisis económica y como efecto de la globalización, sin embargo, también se encontró que se posee un alto potencial de crecimiento, principalmente en cuanto a la destreza y el conocimiento técnico con que cuentan.
Key words

Machining practices; machining industry.

\section{Abstract}

This study of machining practices in Costa Rica details in depth the state of the art of the high precision industry sector, based on the study of representative corporations. The main objective was to identify the practices and establish the research needed to improve them. The results were contrasted with the practices of an international corporation as well as with knowledge obtained from experts of this industry.

Most of the case studies stated that often they find the designs to be missing the part and assembly functionality; hence, they think it would be very useful to enhance people with such knowledge at the university stage. The study also found that very few people in the companies are capable of performing adequate designs.

It was found that despite the recent economic crisis and the globalization side effects, this industry has potential to grow, mainly due to the ability and technical knowledge they demonstrate to have. 


\section{Manufactura de alta precisión}

En la manufactura, los procesos de maquinado han evolucionado considerablemente y eso ha permitido en gran medida la generación e industrialización de mucha de la alta tecnología que actualmente existe, como es el caso de industrias como la automotriz. Los procesos de maquinado varían en cuanto a sus capacidades de precisión, generalmente debido a la capacidad técnica del equipo. Groover (2007) muestra procesos de maquinado bastante precisos. Algunos procesos de maquinado permiten tolerancias de $\pm 0,005 \mathrm{~mm}(0,002$ pulgadas $)$ mientras que otros procesos abrasivos permiten llegar a $\pm 0,0008$ mm (0,0003 pulgadas). La oferta actual de máquinas y herramientas para corte y otros procesos es bastante diversa tanto en capacidades como en costo y durabilidad de las herramientas. Actualmente hay centros de fresado CNC que garantizan exactitudes de $\pm 0,00 \mathrm{Imm}$ (0,00039 pulgadas).

La manufactura de partes o componentes está precedida por el diseño de estos. El diseño es una tarea ingenieril de alta complejidad. El proceso de diseño adquiere relevancia incluso para normas como ISO 9000. La norma ISO 900I:2008, en su séptimo capítulo, concerniente a la realización del producto o servicio, especifica que tanto el diseño como el desarrollo de los productos deben permitir la verificación y ser aprobados antes de la ejecución. Esto obliga a cumplir los requerimientos y proveer información adecuada para la manufactura, indicar los criterios de aceptación y especificar las características esenciales para su uso no solo adecuado sino también seguro. Además, la norma ISO 16949:2002, una norma de los Sistemas de Calidad ISO 9000 con Requerimientos Particulares para la Producción Automotriz, exige que los diseños se expresen en términos que permitan su verificación y validación, así como incluir estudios de análisis de fallas del diseño, conocidos como FMEA, estudios de confiabilidad, prueba y error del producto, especificaciones o características especiales. La validación y la verificación están relacionadas, no obstante, la primera corresponde al fabricante mientras que la segunda atañe al cliente.

El diseño es un proceso en el cual se debe valorar el funcionamiento posterior del componente. Los estándares de los fundamentos para el diseño de productos y procesos, así como de los dibujos técnicos, están definidos en ASME Y I4.5M-1994 y ASME Y 14.5.2-2000GDTP.) La Sociedad Americana de Ingenieros Mecánicos (ASME) califica e incluso acredita a aquellos que se vean involucrados en estas actividades con grados como Profesionales en Dimensionado Geométrico y Tolerancias, comúnmente denotado como GD\&T. Fowlkes y Creveling (1995) indican que la geometría dimensional y tolerancia, GD\&T, es un proceso de comunicación de especificaciones que se enfoca en el desarrollo de un modelo gráfico del diseño después de que el modelo analítico y físico ha sido desarrollado a través de tolerancias. El grado de dificultad que tiene la definición del tipo y dimensionamiento se demuestra en las especificaciones tanto de ASME como de ANSI.

Por lo tanto, establecer tolerancias para las dimensiones de las piezas para su manufactura es una necesidad. Bjorke (1989) indica que es imposible la manufactura de partes exactamente iguales, y más allá tampoco es necesario. McMahon (1998), en sus guías para la simplificación y estandarización en el diseño de productos, indica que los diseñadores deberán procurar evitar el uso de tolerancias ajustadas o acabados de superficie de alta calidad. Guan, Wang y Tao (2009) sugieren la selección de un esquema de manufactura estudiando los costos, tiempos, calidad, niveles de servicio y utilización de recursos así como también reconocer la experiencia y el conocimiento del fabricante.

\section{Método de investigación}

Con el fin de conocer el estado del sector de maquinado de alta tecnología, se estudiaron cuatro empresas nacionales, dos de ellas de las diez citadas por CINDE (20l I) como empresas dedicadas a la manufactura de alta precisión. Las otras dos empresas se seleccionaron por presentar características de interés para el estudio.

Además, se buscó una empresa internacional para comparar con ella los casos nacionales, a sabiendas de la diferencia en los recursos de los que disponen, como personal y equipos de manufactura.

También se consideró relevante para el estudio cruzar algunos resultados con reportes o criterios de conocedores tanto del sector industrial como del sector metalmecánico y de alta precisión en Costa Rica. Así, se buscó a la Cámara de Industrias de Costa Rica, PROCOMER, CINDE, COPRE y LACOMET. 
El principal método de recolección de datos para la investigación fue la visita, durante la cual se pudieron observar los procesos y equipos de fabricación. Además, se entrevistó a los encargados de los diseños, de maquinado e incluso de aseguramiento de la calidad. También fue relevante indagar sobre los métodos para el establecimiento y consecución de los parámetros meta para los productos. Los casos de estudio se caracterizaron por su maquinaria y herramientas, por el conocimiento técnico del personal y por el nivel de precisión que pueden alcanzar. También se estudió el uso de tolerancias, principalmente unilaterales, así como las prácticas de manufactura ante la designación de tolerancias para las dimensiones críticas.

\section{Aspectos relevantes y centrales del estudio de casos}

A continuación, se comparan los resultados de los casos estudiados en cada aspecto relevante.

\section{Sistema de producción}

Los casos estudiados que corresponden a empresas nacionales trabajan al estilo taller con un bajo volumen de una gran variedad de productos. Por el contrario, el caso internacional trabaja un mayor volumen de una menor variedad de productos.

Equipos y maquinaria

Los casos estudiados poseen maquinaria de alta tecnología como CNC, sin embargo, algunos tienen máquinas solo de $\mathrm{CN}$ y en todos también se utilizan equipos convencionales. Uno de los casos estudiados por dedicarse a productos de gran tamaño solo poseía maquinaria convencional. Entre los equipos de más uso destacan las fresadoras, los tornos y las erosionadoras de hilo. Estos equipos y maquinaria varían en cuanto al fabricante, aunque varios dijeron preferir los japoneses.

Los casos estudiados coinciden en que, si bien la precisión final depende de la máquina y de la herramienta, en gran medida también es producto de la habilidad del operador, por lo que afirman que en muchos casos con máquinas convencionales obtienen resultados satisfactorios.

Además, en dos casos incluso se indicó que la maquinaria CNC exige una alta inversión de tiempo para hacer el programa, por lo que no es atractiva para maquinar un bajo volumen de partes.
Medición

Los casos estudiados confirman que se requiere una alta inversión en equipo de medición para poder garantizar el logro de las metas dimensionales establecidas. Este equipo para la manufactura de alta precisión tiene un costo aún mayor.

Todos los casos estudiados poseen equipos y espacios físicos de laboratorio exclusivos para la medición, aunque es importante destacar que se observaron considerables diferencias y en general los laboratorios de las empresas nacionales son los más limitados.

\section{Conocimiento técnico del personal}

En todos los casos estudiados se encontró personal altamente calificado, con alto conocimiento técnico y habilidad práctica. Es importante destacar que la labor del diseño de productos, en todos los casos estudiados, recae en pocas personas. En las empresas nacionales de alta precisión ese personal coincide con ser el propietario. En el caso de la empresa internacional, la labor de diseño la realiza solamente la casa matriz.

\section{Capacidad para alcanzar altas precisiones}

Todos los casos estudiados alcanzan alta o media precisión, según sea la especificación del cliente. Como se ha indicado, varios encargados del maquinado consideran que no se requiere maquinaria altamente especializada, como CNC, para alcanzar en gran medida las dimensiones meta.

Se encontraron diferencias sustanciales en las precisiones alcanzadas, según el tamaño de las partes que producen. Este resultado coincide con la teoría. Así, se encontraron precisiones muy altas, de dos diezmilésimas de pulgada, pero también de dos centésimas de pulgada. En uno de los casos en que se fabrican partes de grandes dimensiones, se considera permisible alcanzar apenas una precisión de una décima de pulgada.

\section{Diseño y maquinado de las partes}

En los casos estudiados se evidenció que el diseño y la manufactura son procesos que se realizan de forma bastante independiente.

La mayoría de los casos estudiados coincide en que, a pesar de las implicaciones que el diseño tiene para la manufactura, siempre se encuentran aspectos que deben mejorarse en los planos o dibujos de 
los diseños de las partes o piezas, pues la mayoría carece de la concepción del funcionamiento final de éstas y por ende de los requerimientos para su acople. Un ejemplo de ello es que muchos planos de partes muestran dimensiones que, una vez acopladas, son difíciles o incluso imposibles de medir.

Los planos de diseño muchas veces se limitan al dibujo de las partes, cuando deberían de considerar adecuadamente el desempeño final. Cuando falta esta consideración, los planos son susceptibles de exigir dimensiones innecesariamente precisas o, por el contrario, de ser excesivamente permisivos en variaciones de dimensiones e incluso carecer de especificaciones de las dimensiones necesarias.

En varios de los casos nacionales, con cierta frecuencia se rediseñan las partes ya que los diseños solicitados por el cliente son demasiado complejos de manufacturar o porque los diseños no se ajustan a los requerimientos del cliente.

La mayoría de los casos estudiados expresa la conveniencia de ampliar el dominio del concepto de la tolerancia del ajuste, para comprender el traslado de esa tolerancia hacia las partes del acople. La mayoría indica que es importante realizar adecuadamente la asignación de tolerancias a las partes por maquinar y sugiere que se debe generar dicho conocimiento desde la formación universitaria de los ingenieros.

\section{Maquinado del valor dimensional meta y uso de tolerancias unilaterales}

En todos los casos, primero se ha diseñado la parte y posteriormente el encargado del maquinado prepara y establece, por su experiencia y habilidad, las condiciones de la maquinaria para poder obtener los valores meta para las dimensiones y otras características de calidad establecidas.

Por otra parte, la práctica del maquinado es un proceso de prueba y error hasta que se logran las dimensiones requeridas o bien unas semejantes que sean satisfactorias. Se encontró que los encargados del maquinado tienden a guiarse poco por el plano, lo cual podría explicarse por las carencias recién expuestas. En algunos de los casos nacionales los planos indicaban las tolerancias, bilaterales o unilaterales, en algunos se adjuntaba una tabla de tolerancias generales, pero en otros solo se indicaba la dimensión meta. En la empresa internacional se constató una mayor guía del plano de diseño en el maquinado; en este caso, el plano posee indicaciones de tolerancias bilaterales o unilaterales según corresponda. Sin embargo, en la etapa de validación se evidenció que en muchos casos el plano de diseño requiere modificaciones en las tolerancias que indica.

\section{Materia prima}

En la mayoría de los casos estudiados se coincide en que se requiere de la importación de materia prima, generalmente acero para sus partes. Solamente dos de las empresas nacionales afirmaron usar materia prima adquirida en Costa Rica. Las otras indicaron que su preferencia por la materia prima importada radica en las características de su calidad.

COPRE recomienda a las empresas mantener relación con laboratorios de materiales, ya que los distintos aceros requieren distintas herramientas, por lo que es conveniente tener conocimiento exacto del acero que se va a trabajar.

\section{Tendencias del mercado}

Los casos de estudio nacionales reportan que, como consecuencia de la globalización, la importación de partes y productos a muy bajo costo ha bajado las demandas de los clientes. También indican que la reciente crisis económica contrajo su producción y obligó a reducir personal. Coinciden en que los precios de venta de algunos productos importados en ocasiones son inferiores al costo de la materia prima para manufacturarlos.

PROCOMER (2010) afirma que el sector metalmecánico se encuentra muy encadenado a empresas transnacionales a las que se les manufacturan piezas y partes. Destaca principalmente el caso de la mecánica de precisión, donde el sector ha demostrado capacidad para diseñar y fabricar piezas y maquinaria a la medida del cliente así como ofrecer servicios de instalación y mantenimiento y reposición de piezas. En la investigación fue posible constatar que este encadenamiento del sector los deja en función del cliente, lo que les afecta negativamente cuando el cliente inesperadamente cesa el consumo del producto. Además, la mayoría de las veces las empresas han debido especializar sus procesos y equipos de taller para estos encadenamientos, por lo que posteriormente, si el cliente cancela sus servicios, será poca la utilidad que tendrán de esas inversiones. 
La mayoría de las empresas investigadas se ha direccionado hacia nuevos mercados, buscando diferenciarse por la calidad de su trabajo. Sin embargo, continúan trabajando al estilo taller, dependiendo de los pedidos de sus clientes, y en ninguna se encontró un producto que implique una producción estable para la empresa.

\section{Estado económico del sector industrial y metalmecánico en Costa Rica}

El índice mensual de actividad económica (IMAE), según el Banco Central de Costa Rica (BCCR) (20I2), decreció en el primer semestre de 2012, demostrando que la industria manufacturera ha tenido una fuerte caída. La encuesta trimestral de la Unión Costarricense de Cámaras y Asociaciones del Sector Empresarial Privado (UCCAEP, 20I2) muestra una caída en el optimismo empresarial como resultado de la creciente incertidumbre por el sistema de bandas cambiarias y la dificultad de acceder a créditos. El BCCR muestra que la tasa real para crédito productivo ha alcanzado los valores máximos de los últimos seis años, incidiendo negativamente en el crecimiento del sector industrial. Estos aspectos dificultan las inversiones y el crecimiento del sector. Caso contrario es el de otros países latinoamericanos que presentan mejores panoramas, como México, Brasil y Chile, donde según Metalmecánica (20/2) más bien se ha observado en la industria un crecimiento por la crisis económica mundial.

La Cámara de Industrias de Costa Rica (20II) considera que actualmente siete factores impiden la competitividad de las industrias, ellos son: costo de la energía eléctrica, precio de las materias primas, sistema cambiario, trámites y permisos, impuestos y cargas sociales, costo de los combustibles y acceso al financiamiento. El financiamiento también lo apunta COPRE como limitante para los pequeños y medianos empresarios, para quienes la inversión inicial para la maquinaria es sumamente difícil y tienen escaso apoyo de la banca.

\section{Discusión y conclusiones}

Los casos estudiados coinciden en que la consecución de los valores de dimensiones meta no se puede delegar exclusivamente en la maquinaria y herramienta utilizada sino que se requiere la habili- dad y el conocimiento del operador. Por otra parte, los proveedores y expertos en maquinaria CNC sostienen que la capacidad que brinda esta tiene mucho impacto y que se debe valorar por la muy alta precisión que permite alcanzar. COPRE indica que es conveniente conocer el potencial de algunas herramientas, ya que se puede requerir su uso en maquinaria más precisa, dado que en otros tipos de maquinaria su rendimiento puede ser inferior.

Los casos nacionales estudiados corresponden a talleres de producción con una alta variedad de partes pero un bajo volumen, por lo que la consecución de los parámetros objetivo, al estar constantemente variando las partes, implica una alta destreza y flexibilidad pero una limitada eficiencia. La programación de la maquinaria CNC para volúmenes de producción bajos puede tomar más tiempo que maquinar la parte; en este escenario, un operador con alto conocimiento y destreza puede preferir maquinar la pieza que realizar el programa. Un escenario muy distinto lo constituyen los procesos productivos de alto volumen de producción.

En todos los casos se evidenció la preocupación por obtener las dimensiones meta. Para la mayoría de los nacionales fue evidente que prevalece un proceso de prueba y error en la manufactura, donde se contrasta la dimensión final resultante de la parte con la especificada. El plano del diseño no siempre actúa como el instrumento que permite guiar la manufactura.

Se observa que aún hay separación entre los procesos de diseño y de manufactura, lo que permite comprender por qué la teoría ha insistido en la necesidad de enlazarlos más. Además, en los casos nacionales se encontró que la labor del diseño recae en muy pocas personas. La mayoría coincide en que los planos requieren de la concepción del funcionamiento final de la parte o de los requerimientos para su acople. Esta concepción es necesaria para la calidad no solo de la parte sino del ensamble final. Por lo tanto, coinciden en la necesidad de tener más dominio en la asignación de tolerancias a las partes. Es conveniente tener más estudios de la teoría del análisis geometría, dimensionamiento y tolerancias (GD\&T), que se ha desarrollado para potenciar el adecuado diseño y que sería de gran utilidad para la manufactura a nivel nacional.

En los casos nacionales se encontró capacidad e inventiva para el diseño de partes e incluso de 
maquinaria. Convendría fortalecer y estimular el desarrollo de esa capacidad, así como promover el interés por manufacturar productos propios.

Los casos nacionales trabajan en un estilo de producción de taller, dependientes de la demanda del cliente. En ningún caso se encontró un producto que implique una producción estable para la empresa. En una de las dos empresas nacionales estudiadas se constato el perjudicial impacto económico que se puede experimentar cuando el cliente al que principalmente se está encadenado prescinde de sus servicios. Por lo tanto, abordar los encadenamientos se convierte en un aspecto crucial para el sector.

Los estudios de análisis de fallas (FMEA), requisito de normas como ISO 900 I- 2008, solo se constataron en la empresa internacional. Los casos nacionales carecen de certificaciones, donde la barrera de entrada es el alto costo, pero la ausencia de estas les coloca en una posición de desventaja.

COPRE considera necesario consolidar los sistemas de medición. LACOMET también apunta a la medición como limitante para el sector industrial, principalmente las Pymes, e indica que la medición del cumplimiento de las dimensiones requiere de instrumentación precisa y métodos de medición regulados, destacando también que se necesita una adecuada calibración de los equipos.

El sector metalmecánico de alta precisión en Costa Rica ha sido golpeado por la reciente crisis económica. La creciente importación de productos maquinados en otros países en grandes volúmenes y vendidos a bajo costo, en contrate con el incremento en los costos de las materias primas y de la energía, han incidido negativamente. Por el contrario, la industria metalmecánica ha crecido en los últimos años en México y en Argentina.

En la investigación se encontró que las empresas nacionales cuentan con personal de alto conocimiento y destreza técnica, por lo que mediante una estrategia de competitividad y mercadeo, así como de una mejora en las prácticas de diseño y maquinado, podrían lograr más estabilidad y apuntar a un crecimiento más promisorio.

De la investigación se concluye que las empresas nacionales podrían lograr más estabilidad y apuntar a un crecimiento más promisorio, mediante una mejora en las prácticas de diseño y maquinado así como una estrategia de competitividad y mercadeo, ya que cuentan con personal de alto conocimiento y destreza técnica.

\section{Bibliografía}

BCCR (Banco Central de Costa Rica). (20/2). Indicadores Económicos del Banco Central de Costa Rica. Recuperado el II de agosto de 2012 de: http://indicadoreseconomicos. bccr.fi.cr/indicadoreseconomicos/Documentos/Informe\%20 Mensual/INFORME\%20MENSUAL\%2020 I 2/Informe\%20 mensual\%20de\%20Coyuntura\%20Econ\%C3\%B3mica,\%20 \%20mayo\%2020 I 2.pdf

Bjorke, O. (1989). Computer Aided Tolerancing. 2 ed. Asme Press.

Cámara de Industrias de Costa Rica. (2011). Sector industrial sufre desaceleración en el 2011 y augura un 2012 lleno de retos. Recuperado del sitio web de Cámara de Industrias de Costa Rica: http://www.cicr.com/index. php?option=com_content\&view=article\&id =540:segunbalance-anual-de-la-camara-de-industrias-sector-industrialsufre-desaceleracion-en-el-20 I I-y-augura-un-20 I 2-Ilenode-retos\&catid $=441$ : comunicados-de-prensa\&ltemid $=3$

CINDE (Coalición Costarricense de Iniciativas de Desarrollo). (20I I). Base de Proveedores de Costa Rica. Recuperado el 8 de junio del 2012 de www.cinde.org

Fowlkes, W.Y. \& Creveling, C.M. (1995). Engineering Methods for Robust Product Design. Engineering Process Improvement Series.

Guan, X., Wang, Y. \& Tao, L. (2009). Machining scheme selection of digital manufacturing based on genetic algorithm and AHP. Journal of intelligent manufacturing Vol. 20. N 6 . Recuperado el 20 de febrero del 2012 de SPRINGER LINK

Groover, M. (2007). Fundamentos de Manufactura Moderna: Materiales, Procesos y Sistemas. McGraw Hill.

McMahon C. \& Browne J. (1998). CAD CAM Principles, practice and manufacturing management. 2 ed. Addison Wesley Longman.

Metalmecánica. (2012). La industria metalmecánica de cara a 2012. Recuperado el 8 de agosto de 2012 de: http:// www.metalmecanica.com/mm/secciones/MM/ES/MAIN/ IN/INFORMES_ESPECIALES/doc_86569_HTML. html?idDocumento $=86569$

PROCOMER (Promotora de Comercio Exterior). (2010). Diversificando las exportaciones metalmecánicas hacia Panamá. Recuperado el 6 de setiembre 2012 de: http:// wvw.elfinancierocr.com/ef_archivo/2010/noviembre/07/_ MMedia/00000 I0684.pdf

UCCAEP (Unión Costarricense de Cámaras y Asociaciones del Sector Empresarial Privado). (2012). Encuesta Trimestral: Caída de indicadores de percepción empresarial. Recuperado el 24 de agosto de 2012 de: http://www.uccaep.or.cr/index. php/sala-de-prensa/noticias/26-indicadores-empresarialesmuestran-fuerte-desaceleracion 